\title{
Salvianolic acids: small compounds with multiple mechanisms for cardiovascular protection
}

Jennifer Hui-Chun $\mathrm{Ho}^{1,2,3}$ and Chuang-Ye Hong ${ }^{1,4^{*}}$

\begin{abstract}
Salvianolic acids are the most abundant water-soluble compounds extracted from Radix Salvia miltiorrhiza (Danshen). In China, Danshen has been wildly used to treat cardiovascular diseases for hundreds of years. Salvianolic acids, especially salvianolic acid A (Sal A) and salvianolic acid B (Sal B), have been found to have potent anti-oxidative capabilities due to their polyphenolic structure. Recently, intracellular signaling pathways regulated by salvianolic acids in vascular endothelial cells, aortic smooth muscle cells, as well as cardiomyocytes, have been investigated both in vitro and in vivo upon various cardiovascular insults. It is discovered that the cardiovascular protection of salvianolic acids is not only because salvianolic acids act as reactive oxygen species scavengers, but also due to the reduction of leukocyte-endothelial adherence, inhibition of inflammation and metalloproteinases expression from aortic smooth muscle cells, and indirect regulation of immune function. Competitive binding of salvianolic acids to target proteins to interrupt protein-protein interactions has also been found to be a mechanism of cardiovascular protection by salvianolic acids. In this article, we review a variety of studies focusing on the above mentioned mechanisms. Besides, the target proteins of salvianolic acids are also described. These results of recent advances have shed new light to the development of novel therapeutic strategies for salvianolic acids to treat cardiovascular diseases.
\end{abstract}

\section{Introduction}

Salvianolic acid is one of the bioactive compounds of S. miltiorrhiza BGE extracted from the root of S. miltiorrhiza, commonly named "Danshen" in China. According to traditional Chinese medicine, Danshen can be used to promote blood flow and to resolve blood stasis. Therefore, it is wildly prescribed to patients with angina pectoris, hyperlipidemia, and acute ischemic stroke [1-3]. Using chromatographic fingerprinting method and mass spectrometry, there are more than eighteen components in Radix S. miltiorrhiza. They can be classified as watersoluble (hydrophilic) phenolic compounds and nonpolar (lipid-soluble) diterpenoidal compounds [4,5]. Salvianolic acids are the main water-soluble compound in S. miltiorrhiza. Among salvianolic acids, Sal A and Sal B are the most abundant components. The structures of salvianolic acids are shown in figure 1.

The bioavailability, pharmacodynamics as well as pharmacokinetics of salvianolic acids have been investigated.

\footnotetext{
* Correspondence: hongprof@tmu.edu.tw

'Graduate Institute of Clinical Medicine, Taipei Medical University, Taipei,

Taiwan

Full list of author information is available at the end of the article
}

Due to first-order absorption, Sal B reaches the maximum plasma concentration within 0.5-1 hour and could be detected up to 180 minutes after oral administration $[6,7]$, and which undergoes hepatobiliary excretion [8]. In conscious and freely moving rat, $\mathrm{Wu}$ et al. demonstrates that Sal B shows the linearity over a plasma concentration range of $0.5-200 \mu \mathrm{g} / \mathrm{ml}$ and $83.78 \pm 10.5 \%$ of plasma protein binding rate. By intravenous injection, $100 \mathrm{mg} / \mathrm{kg}$ Sal B reaches the maximal plasma concentration $\left(C_{\max }\right)$ around $910 \mu \mathrm{g} / \mathrm{ml}$, and the half life $\left(t_{1 / 2}\right)$ of Sal B is around 105 minutes [7]. For oral administration, the $C_{\max }$ of $500 \mathrm{mg} / \mathrm{kg} \mathrm{Sal} \mathrm{B} \mathrm{is} 1.5 \mu \mathrm{g} / \mathrm{ml}$ while $100 \mathrm{mg} / \mathrm{kg}$ Sal A is only $308 \mathrm{ng} / \mathrm{ml}$; the $t_{1 / 2}$ is around 248 minutes for Sal B and 3.29 hours for Sal A [7,9]. The oral bioavailability of Sal B in a conscious rat is calculated to be $2.3 \%$ [7], which is higher than rats under general anesthesia reported by Zhang et al [10].

Although the therapeutic potential of salvianolic acids on hepatic protection [11,12], neural protection [13,14], and cancer treatment [15-17] have been proposed in recent years, the greatest clinical impact of salvianolic acids is cardiovascular protection. In the past few years, mechanism(s) of how salvianolic acids regulate endothelial

\section{() Biomed Central}


<smiles>O=C(/C=C/c1ccc(O)c(O)c1/C=C/c1ccc(O)c(O)c1)OC(Cc1ccc(O)c(O)c1)C(=O)O</smiles>

Salvianolic acid A<smiles>O=C(O)/C=C/c1ccc(O)c2c1C(C(=O)OCC(=O)OC(Cc1ccc(O)c(O)c1)C(=O)O)[C@H](c1ccc(O)c(O)c1)O2</smiles>

Salvianolic acid B
Molecular Formula: $\mathrm{C}_{26} \mathrm{H}_{22} \mathrm{O}_{10}$ Molecular Weight: 494.45

\author{
Molecular Formula: $\mathrm{C}_{36} \mathrm{H}_{30} \mathrm{O}_{16}$ \\ Molecular Weight: 718.6138
}

Figure 1 Chemical structure of salvianolic acid A (Sal A) and Sal B. More than eighteen components can be identified in Radix S. miltiorrhiza. Sal B is the most abundant while Sal A is the most potent water-soluble phenolic component in Radix S. miltiorrhiza.

cells, vascular smooth muscle cells and cardiomyocytes have been investigated. In this article, we summarize results of these studies on the cardiovascular protective effect of salvianolic acids and elucidate the multiple mechanisms of these small compounds in terms of reactive oxygen species (ROS) scavenging ability, leukocyteendothelial adhesion regulation, inflammation inhibition and immune-modulation. In addition, intracellular signaling pathway regulated by salvianolic acids as well as putative proteins targeted by salvianolic acids are described in this article.

\section{Cardiovascular Peotection of Salvianolic Acids}

A.

\section{Salvianolic Acids Serve As Potent Ros Scavengers During} Cardiovascular Injury

Due to their polyphenolic structure, salvianolic acids are thought to be free radical scavengers. Indeed, both Sal B and Sal A show their high radical scavenging capacity measured by neutralizing free radicals assays such as DPPH radical scavenging test or ABTS assay [18-20]. Liu et al reported that seven phenolic compounds isolated from S. miltiorrhiza inhibited lipid peroxidation of rat liver microsomes induced by iron/cysteine and vitamin $\mathrm{C} / \mathrm{NADPH}$ and the hemolysis of rat erythrocytes induced by $\mathrm{H}_{2} \mathrm{O}_{2}$ in vitro [21]. It was found that Sal A was the most potent antioxidant among the salvianolic acids. However, Sal B was thought to have much more commercial value for the food and medicine purposes due to the containment of the highest amounts in $S$. miltiorrhiza [4,18,21]. Sal B exhibited higher scavenging activities than vitamin $\mathrm{C}$ against $\mathrm{HO}, \mathrm{O} 2^{--}, \mathrm{DPPH}$ radicals, and ABTS radicals. However, their iron chelating and $\mathrm{H}_{2} \mathrm{O}_{2}$ scavenging activities were lower than vita$\min C[20]$.

Salvianolic acids were not only demonstrated to have antioxidant activity in vitro, but also been proven to act as cardiovascular protectors in vivo. Using ischemiareperfusion injury model of an isolated rat heart, it was demonstrated that Sal A lowered the ventricular fibrillation rate, decreased cellular LDH leaking and reduced lipid peroxidation in damaged cardiac tissue [22]. Wu and Hong et al reported that feeding with 5\% watersoluble extract of $S$ miltiorrhiza which contained Sal B significantly lowered plasma cholesterol level, reduced endothelial damage and the severity of atherosclerosis in diet-induced hypercholesteremic rabbits. The cardiovascular protection potential of Sal B was contributed by its ROS scavenging ability. Sal B-treated LDL exhibited vitamin E-binding ability and was resistant to $\mathrm{Cu}^{2}$ ${ }^{+}$-induced oxidation [23]. Moreover, intravenous administration of Sal A $(0.3-3 \mathrm{mg} / \mathrm{kg})$ significantly attenuated isoproterenol-induced cardiac dysfunction and myocardial injury, and improved mitochondrial respiratory function in rat with isoproterenol-induced myocardial infarction [24].

It is known that increase in oxidative stress induced the proliferation of aortic smooth muscle cells. Recently, salvianolic acids were found to inhibit the proliferation of rat aortic smooth muscle A10 cells stimulated by homocysteine, an oxidative stress factor. Elucidation of proteomic changes by two-dimensional electrophoresis coupled with MALDI-TOF mass spectrometry revealed that the inhibitory effect of the salvianolic acid on 
homocysteine-induced A10 cell proliferation was via the $\mathrm{PKC} / \mathrm{p} 44 / 42$ MAPK dependent pathway [25]. Interestingly, salvianolic acid treatment reduces the carbonylation of specific cytoskeleton and chaperone proteins such as vimentin, $\alpha 4$-tropomyosin and GRP75, and lead to phenotype transformations in the rat A10 cells [25].

Apart from what have been mentioned above, salvianolic acids have been reported to protect cardiomyocytes from drug-induced toxicity due to its ROS scavenging ability. It was noted that Sal A converted $\mathrm{HO}$ generated by electron transfer from adriamycin semiquinone radicals to $\mathrm{H}_{2} \mathrm{O}_{2}$ on adriamycin-induced mitochondrial toxicity of rat heart in a dose-dependent manner [26]. In mice with doxorubicin-induced cardiotoxicity, salvianolic acids (containing 64.92\% Sal B, 40 $\mathrm{mg} / \mathrm{kg} /$ day for 3 days) also protected myocardium through reducing oxidative stress [27].

B.

\section{Salvianolic Acids Inhibit Leukocyte-Endothelial Cell Adherence}

Leukocyte attachment, migration as well as adhesion molecule expression on arterial endothelial cells all are important steps in the development of early atherosclerosis. A series of studies on salvianolic acids regulating leukocyte-endothelial cell adherence have been undertaken. It is well-established that endothelial-leukocyte adhesion molecules on aortic endothelial cells can be induced by TNF- $\alpha$. Sal B was found to attenuate VCAM-1 and ICAM-1, but not E-selectin expression, in a dose-dependent manner (1-20 $\mu \mathrm{g} / \mathrm{ml})$, in TNF- $\alpha$-treated HAECs. These effects were associated with its anti-inflammatory property through inhibiting the activation of NFkB pathway triggered by TNF- $\alpha$ [28].

Using Evans Blue dye-labeled bovine serum albumin to investigate the alteration of permeability in HUVECs caused by Sal B $(20-50 \mu \mathrm{g} / \mathrm{ml})$, it was found that Sal B reduced the permeability and attenuated the disorganization of VE-cadherin induced by TNF- $\alpha$ in these cells. The effect was attributed to the reduction of VEGF protein expression as a result of modulation of the ERK pathway [29]. Loss of cell-cell adhesion junctions also increased endothelial permeability. Dang et al demonstrated the protective effect of Sal B on TNF- $\alpha$-mediated disorganization of endothelial cell junctions through attenuating tyrosine phosphorylation of cell junction proteins such as VE-cadherin and $\beta$-catenin. Results of immuno-precipitation studies indicated that Sal B prevented $\beta$-catenin disassociation from the cytoskeleton in TNF- $\alpha$-treated HUVECs [30].

Zhou et al reported that treatment with Sal B (0.05 and $0.15 \mu \mathrm{M})$ significantly inhibited PAI-1 gene expression in the first 18 hours when HUVECs were exposed to TNF- $\alpha$. It was further demonstrated that NFkB and
ERK-AP-1 pathways were possible targets of Sal B in regulating TNF- $\alpha$-stimulated PAI-1 production in HUVECs [31].

\section{C.}

Salvianolic Acids Inhibit Inflammation And Regulation of Metalloproteinaseses Expression During Cardiovascular Injury

Synthesis and release of inflammatory cytokines from vascular smooth muscle cells is an important contributor to the pathogenesis of atherosclerosis. Chen et al and Lin et al reported respectively that genetic expression of COX-2 and protein expression of MMP-2 and MMP-9 in LPS-treated HASMCs could be inhibited by Sal B via the suppression of ERK1/2 and JNK phosphorylation, reduction of PGE2 production and NADPH oxidase activity [32,33]. In ApoE-deficient mice fed with high cholesterol diet, supplementation with $0.3 \%$ of Sal $B$ protected mice from atherosclerosis by reducing the thickness of intima, which was accompanied by a significant reduction of COX-2, MMP-2 and MMP-9 expression [32,33].

Sal B not only inhibited MMP-2 activation induced by LPS, but also inhibited MMP-2 activation induced by TNF- $\alpha$, angiotension II and $\mathrm{H}_{2} \mathrm{O}_{2}$. It has been demonstrated that Sal B inhibited TNF- $\alpha$, angiotension II and $\mathrm{H}_{2} \mathrm{O}_{2}$-induced MMP-2 mRNA, protein expression, and gelatinolytic activity in HASMCs in a concentrationdependent manner (0.1-10 $\mu \mathrm{M})$, which was through the inhibition of NADPH oxidase-dependent ROS generation [34].

In experimental myocardial infarction in rat, Jiang et al reported that administration of salvianolic acids significantly decreased infarct size, improved left ventricular function and decreased myocardial malondialdehyde levels compared with the control group. The cardioprotection of salvianolic acids against infarct-induced left ventricle remodeling was significantly contributed by the down-regulation of MMP-9 mRNA expression level and its activity at the infarct area [35]. With molecular modeling, in-gel gelatin zymography and enzymatic analysis, Jiang et al demonstrated that Sal B (from 0-70 $\mu \mathrm{M}$ ) bound to MMP-9 at catalytic domain and functioned as a competitive inhibitor of MMP-9 [36].

D.

\section{Salvianolic Acids Regulate Kinase Activity and are Potential Immunomodulators}

Recently, the putative protein targets of salvianolic acids have been investigated [37]. In addition to MMP-9 binding affinity [36], salvianolic acids regulate intracellular kinaseassociated signaling pathway [28,29,31-34], indicating that salvianolic acids interact with phosphotyrosine or phosphoserine/threonine-binding domain [38,39]. With binding 
affinity assay and molecular modeling prediction, Sperl et al reported that Sal A and Sal B were inhibitors of the protein-protein interaction mediated by SH2 domains of Srcfamily kinases Src and Lck. The potency of Sal A and Sal B (from 0-100 $\mu \mathrm{M}$ ) binding to Src and Lck were higher than rosmarinic acid, a nature product known as a Lck SH2 domain inhibitor [40]. Since Lck is a T cell-restricted Src family protein tyrosine kinase and is crucial in TCRmediated signaling pathway, the activity of rosmarinic acid against Lck SH2 domain has been used experimentally as an immune-suppressive agent [41] and becomes a focus of cancer drug discovery [42].

Recently, Wang et al reported that using an ELISAlike HTS assay, Sal B and rosmarinic acid were found to be active compounds showing high affinity against CD36, a high affinity receptor for oxLDL, thus prevented oxLDL from macrophage uptake [43]. Since both Sal A and Sal B shared the core structure of rosmarinic acid [40], the high affinity to SH2 domains of Src-family kinases and CD36 suggested the role of immune modulator in the cardiovascular protective effect of salvianolic acids.

\section{Conclusion}

Salvianolic acids, which contain polyphenolic structure, are potent antioxidants. Salvianolic acids reduce intracellular as well as intravascular oxidative stress, which protects endothelial cells, arterial smooth muscle cells, cardiomyocyte, and LDL form free radical damage and peroxidation. In addition, salvianolic acids attenuate endothelial-leukocyte adhesion molecules expression on vascular endothelial cells through regulating intracellular kinase activity. Such kinase-associated signaling pathway inhibition by salvianolic acids also contributes their antiinflammation effect. Salvianolic acids possess strong affinity to bind MMP-9, SH2 domain of the Src-family kinases and CD36, which inhibits protein-protein interaction. For clinical application, intravenous injection rather than oral administration of such a water-soluble compound is more easily to reach the therapeutic plasma concentration. Taken together, the cardiovascular protective effect of salvianolic acids is mediated through multiple molecular mechanisms. Such unique property makes salvianolic acids excellent candidates for future development of cardiovascular protective agents.

\section{List of abbreviations}

S. miltiorrhiza $=$ Salvia miltiorrhiza; Sal $A=$ salvianolic acid $A ;$ Sal $B=$ salvianolic acid $B ;$ ROS = reactive oxygen species; DPPH =1,1-diphenyl-2picrylhydrazyl; $A B T S=2,2$-azino-bis-(3-ethylbenzothiazoline-6-sulfonic acid; $\mathrm{NADPH}=$ nicotinamide adenine dinucleotide phosphate; $\mathrm{H}_{2} \mathrm{O}_{2}=$ hydrogen peroxide; $\mathrm{HO}=$ free hydroxyl radicals; $\mathrm{O}^{--}=$superoxide anion radicals; $\mathrm{LDH}=$ lactate dehydrogenase; LDL = low-density lipoprotein; MALDI-TOF = matrix assisted laser desorption ionization- time of flight; PKC = protein kinase $C_{\text {; }}$ MAPK = mitogen-activated protein kinase; a4-tropomyosin = alpha-4- tropomyosin; GRP75 = glucose regulated protein 75; TNF- $a=$ tumor necrosis factor-alpha; VCAM-1 = vascular adhesion molecule-1; ICAM-1 = intercellular cell adhesion molecule-1; E-selectin = endothelial cell selectin; HAECs = human aortic endothelial cells; NFkB = nuclear factor kappa B; HUVECs = umbilical vein endothelial cells; VE-cadherin = vascular endothelial cadherin VEGF = vascular endothelial growth factor; ERK = extracellular signalregulated kinase; $\beta$-catenin = beta-catenin; PAI-1 = plasminogen activator inhibitor type 1; AP-1 = activating protein-1; COX-2 = cyclooxygenase-2; MMP = metalloproteinases; LPS = lipopolysaccharide; HASMCs = human aortic smooth muscle cells; JNK = c-Jun N-terminal kinases; PGE2 = prostaglandin E2; $\mathrm{ApoE}=$ apolipoprotein $\mathrm{E} ; \mathrm{SH} 2=\mathrm{SrCH}$ Homology 2; Lsk = lymphocyte-specific protein tyrosine kinase; $T C R=T$ cell receptor; ELISA = enzyme-linked immunosorbent assay; HTS = high-throughput screening; oxLDL = oxidized low-density lipoprotein; $C D=$ cluster of differentiation.

\section{Acknowledgements}

The authors acknowledge Professor Oscar K. Lee for proof reading of this manuscript. The authors also acknowledge the financial supports from the Wan Fang Hospital, Taipei Medical University (99TMU-WFH-01-4 and 100 swf03 to $\mathrm{JHH}$ ), as well as the support of research grants from the National Science Council (NSC98-2314-B-038-010 and NSC99-2120-M-010001, to $J H H)$.

\section{Author details}

${ }^{1}$ Graduate Institute of Clinical Medicine, Taipei Medical University, Taipei, Taiwan. ${ }^{2}$ Department of Ophthalmology, Wan Fang Hospital, Taipei Medical University, Taipei, Taiwan. ${ }^{3}$ Center for Stem Cell Research, Wan Fang Hospital, Taipei Medical University, Taipei, Taiwan. ${ }^{4}$ Department of Medicine, Wan Fang Hospital, Taipei Medical University, Taipei, Taiwan.

\section{Authors' contributions}

$\mathrm{JH}$ carried out the design, acquisition, analysis and interpretation of data, drafting the manuscript. CY had contributed to conception, design and critical version of important intellectual content and final approval of the manuscript.

\section{Authors' information}

Dr. Jennifer Hui-Chun Ho is the Director of Center for Stem Cell Research and the Deputy Director of Medical Research and Education at Wan Fang Hospital, Taipei Medical University, and is also an Assistant Professor at Graduate Institute of Clinical Medicine, Taipei Medical University. Her main research theme is translational research of stem cells, especially stem cell transplantation.

Prof. Chuang-Ye Hong is the Superintendent of Wan Fang Hospital, Taipei Medical University and also a consultant cardiologist at Wan Fang Hospital. Prof. Hong is an expert in pharmacological research of medical herbs and cardiovascular diseases. Prof. Hong was the Director of Institute of Traditional Medicine at National Yang-Ming University from 1992 to 1997, when he led a research team working on translational research of traditional Chinese medicine, especially Danshen and magnolol.

\section{Competing interests}

The authors declare that they have no competing interests.

Received: 18 February 2011 Accepted: 11 May 2011

Published: 11 May 2011

\section{References}

1. Xu RS: Danshen-biology and its applications Beijing: Scientific Press; 1990

2. Zhou L, Zuo Z, Chow MS: Danshen: an overview of its chemistry, pharmacology, pharmacokinetics, and clinical use. J Clin Pharmacol 2005, 45:1345-1359.

3. Wang X, Morris-Natschke SL, Lee KH: New developments in the chemistry and biology of the bioactive constituents of Tanshen. Med Res Rev 2007, 27:133-148.

4. Hu P, Liang QL, Luo GA, Zhao ZZ, Jiang ZH: Multi-component HPLC fingerprinting of Radix Salviae Miltiorrhizae and its LC-MS-MS identification. Chem Pharm Bull (Tokyo) 2005, 53:677-683.

5. Liu M, Li YG, Zhang F, Yang L, Chou GX, Wang ZT, Hu ZB: Chromatographic fingerprinting analysis of Danshen root (Salvia miltiorrhiza Radix et Rhizoma) and its preparations using high 
performance liquid chromatography with diode array detection and electrospray mass spectrometry (HPLC-DAD-ESI/MS). J Sep Sci 2007, 30:2256-2267.

6. Zhang J, Yu H, Sheng Y, Li L, Ye M, Guo D: HPLC determination and pharmacokinetic studies of salvianolic acid B in rat plasma after oral administration of Radix Salviae Miltiorrhizae extract. Biomed Chromatogr 2005, 19:15-18

7. Wu YT, Chen YF, Hsieh YJ, Jaw I, Shiao MS, Tsai TH: Bioavailability of salvianolic acid B in conscious and freely moving rats. Int J Pharm 2006, 326:25-31

8. Chen YF, Jaw I, Shiao MS, Tsai TH: Determination and pharmacokinetic analysis of salvianolic acid $B$ in rat blood and bile by microdialysis and liquid chromatography. J Chromatogr A 2005, 1088:140-145.

9. Pei L, Bao Y, Wang H, Yang F, Xu B, Wang S, Yang X, Du G: A sensitive method for determination of salvianolic acid $A$ in rat plasma using liquid chromatography/tandem mass spectrometry. Biomed Chromatogr 2008, 22:786-794

10. Zhang Y, Akao T, Nakamura N, Duan CL, Hattori M, Yang XW, Liu JX: Extremely low bioavailability of magnesium lithospermate $B$, an active component from Salvia miltiorrhiza, in rat. Planta Med 2004, 70:138-142.

11. Yan X, Zhou T, Tao Y, Wang Q, Liu P, Liu C: Salvianolic acid B attenuates hepatocyte apoptosis by regulating mediators in death receptor and mitochondrial pathways. Exp Biol Med (Maywood) 2010, 235:623-632.

12. Tsai MK, Lin YL, Huang YT: Effects of salvianolic acids on oxidative stress and hepatic fibrosis in rats. Toxicol Appl Pharmacol 2010, 242:155-164.

13. Chen T, Liu W, Chao X, Zhang L, Qu Y, Huo J, Fei Z: Salvianolic acid B attenuates brain damage and inflammation after traumatic brain injury in mice. Brain Res Bull.

14. Wang SX, Hu LM, Gao XM, Guo H, Fan GW: Anti-inflammatory activity of salvianolic acid $B$ in microglia contributes to its neuroprotective effect. Neurochem Res 2010, 35:1029-1037.

15. Zhou ZT, Yang Y, Ge JP: The preventive effect of salvianolic acid B on malignant transformation of DMBA-induced oral premalignant lesion in hamsters. Carcinogenesis 2006, 27:826-832.

16. Hao Y, Xie T, Korotcov A, Zhou Y, Pang X, Shan L, Ji H, Sridhar R, Wang P, Califano J, Gu X: Salvianolic acid B inhibits growth of head and neck squamous cell carcinoma in vitro and in vivo via cyclooxygenase- 2 and apoptotic pathways. Int J Cancer 2009, 124:2200-2209.

17. Liu X, Yang Y, Zhang X, Xu S, He S, Huang W, Roberts MS: Compound Astragalus and Salvia miltiorrhiza extract inhibits cell invasion by modulating transforming growth factor-beta/Smad in HepG2 cell. $J$ Gastroenterol Hepatol 2010, 25:420-426.

18. Sun Y, Zhu H, Wang J, Liu Z, Bi J: Isolation and purification of salvianolic acid $A$ and salvianolic acid $B$ from Salvia miltiorrhiza by high-speed counter-current chromatography and comparison of their antioxidant activity. J Chromatogr B Analyt Technol Biomed Life Sci 2009, 877:733-777.

19. Zhao GR, Zhang HM, Ye TX, Xiang ZJ, Yuan YJ, Guo ZX, Zhao LB: Characterization of the radical scavenging and antioxidant activities of danshensu and salvianolic acid B. Food Chem Toxicol 2008, 46:73-81.

20. Zhao GR, Zhang HM, Ye TX, Xiang ZJ, Yuan YJ, Guo ZX, Zhao LB: Characterization of the radical scavenging and antioxidant activities of danshensu and salvianolic acid B. Food Chem Toxicol 2008, 46:73-81.

21. Liu GT, Zhang TM, Wang BE, Wang YW: Protective action of seven natural phenolic compounds against peroxidative damage to biomembranes. Biochem Pharmacol 1992, 43:147-152.

22. Du GH, Oiu Y, Zhang JT: Protective effect of salvianolic acid a on ischemia-reperfusion induced injury in isolated rat heart. Yao Xue Xue Bao 1995, 30:731-735.

23. Wu YJ, Hong CY, Lin SJ, Wu P, Shiao MS: Increase of Vitamin E Content in LDL and Reduction of Atherosclerosis in Cholesterol-Fed Rabbits by a Water-Soluble Antioxidant-Rich Fraction of Salvia miltiorrhiza. Arterioscler Thromb Vasc Biol 1998, 18:481-486.

24. Wang SB, Tian S, Yang F, Yang HG, Yang XY, Du GH: Cardioprotective effect of salvianolic acid $A$ on isoproterenol-induced myocardial infarction in rats. Eur \& Pharmacol 2009, 615:125-132.

25. Hung YC, Wang PW, Pan TL, Bazylak G, Leu YL: Proteomic screening of antioxidant effects exhibited by radix Salvia miltiorrhiza aqueous extract in cultured rat aortic smooth muscle cells under homocysteine treatment. J Ethnopharmacol 2009, 124:463-474.
26. Lin TJ, Liu GT, Liu Y, Xu GZ: Protection by salvianolic acid A against adriamycin toxicity on rat heart mitochondria. Free Radic Biol Med 1992, 12:347-351.

27. Jiang B, Zhang L, Li M, Wu W, Yang M, Wang J, Guo DA: Salvianolic acids prevent acute doxorubicin cardiotoxicity in mice through suppression of oxidative stress. Food Chem Toxicol 2008, 46:1510-1515.

28. Chen YH, Lin SJ, Ku HH, Shiao MS, Lin FY, Chen JW, Chen YL: Salvianolic acid B attenuates VCAM-1 and ICAM-1 expression in TNF-alpha-treated human aortic endothelial cells. J Cell Biochem 2001, 82:512-521.

29. Ding M, Ye TX, Zhao GR, Yuan YJ, Guo ZX: Aqueous extract of Salvia miltiorrhiza attenuates increased endothelial permeability induced by tumor necrosis factor-alpha. Int Immunopharmacol 2005, 5:1641-1651.

30. Ding M, Yuan YJ: Study on the mechanisms of an extract of Salvia miltiorrhiza on the regulation of permeability of endothelial cells exposed to tumour necrosis factor-alpha. J Pharm Pharmacol 2007, 59:1027-1033.

31. Zhou Z, Liu Y, Miao AD, Wang SQ: Salvianolic acid B attenuates plasminogen activator inhibitor type 1 production in TNF-alpha treated human umbilical vein endothelial cells. J Cell Biochem 2005, 96:109-116.

32. Chen YL, Hu CS, Lin FY, Chen YH, Sheu LM, Ku HH, Shiao MS, Chen JW, Lin SJ: Salvianolic acid B attenuates cyclooxygenase-2 expression in vitro in LPS-treated human aortic smooth muscle cells and in vivo in the apolipoprotein-E-deficient mouse aorta. J Cell Biochem 2006, 98:618-631.

33. Lin SJ, Lee IT, Chen YH, Lin FY, Sheu LM, Ku HH, Shiao MS, Chen JW, Chen YL: Salvianolic acid B attenuates MMP-2 and MMP-9 expression in vivo in apolipoprotein-E-deficient mouse aorta and in vitro in LPStreated human aortic smooth muscle cells. J Cell Biochem 2007. 100:372-384

34. Zhang HS, Wang SQ: Salvianolic acid B from Salvia miltiorrhiza inhibits tumor necrosis factor-alpha (TNF-alpha)-induced MMP-2 upregulation in human aortic smooth muscle cells via suppression of $\mathrm{NAD}(\mathrm{P}) \mathrm{H}$ oxidasederived reactive oxygen species. J Mol Cell Cardiol 2006, 41:138-148.

35. Jiang B, Wu W, Li M, Xu L, Sun K, Yang M, Guan S, Liu X, Guo DA Cardioprotection and matrix metalloproteinase- 9 regulation of salvianolic acids on myocardial infarction in rats. Planta Med 2009, 75:1286-1292.

36. Jiang B, Chen J, Xu L, Gao Z, Deng Y, Wang Y, Xu F, Shen X, Guo DA: Salvianolic acid $B$ functioned as a competitive inhibitor of matrix metalloproteinase- 9 and efficiently prevented cardiac remodeling. BMC Pharmacol 2010, 10:10.

37. Liu S, Sun Y, Chen H, Song S, Xu Y: Magnetic screening of the potential targeted protein of salvianolic acid B using $\mathrm{T7}$ phage display library. Appl Biochem Biotechnol 2010, 162:1206-1213.

38. Schlessinger J, Lemmon MA: SH2 and PTB domains in tyrosine kinase signaling. Sci STKE 2003 2003, RE12.

39. Yaffe MB, Smerdon SJ: The use of in vitro peptide-library screens in the analysis of phosphoserine/threonine-binding domain structure and function. Annu Rev Biophys Biomol Struct 2004, 33:225-244.

40. Sperl B, Seifert MH, Berg T: Natural product inhibitors of protein-protein interactions mediated by Src-family SH2 domains. Bioorg Med Chem Lett 2009, 19:3305-3309.

41. Won J, Hur YG, Hur EM, Park SH, Kang MA, Choi Y, Park C, Lee KH, Yun Y. Rosmarinic acid inhibits TCR-induced T cell activation and proliferation in an Lck-dependent manner. Eur J Immunol 2003, 33:870-879.

42. Shakespeare WC: SH2 domain inhibition: a problem solved? Curr Opin Chem Biol 2001, 5:409-415.

43. Wang L, Bao Y, Yang Y, Wu Y, Chen X, Si S, Hong B: Discovery of antagonists for human scavenger receptor CD36 via an ELISA-like highthroughput screening assay. J Biomol Screen 2010, 15:239-250.

doi:10.1186/1423-0127-18-30

Cite this article as: $\mathrm{Ho}$ and Hong: Salvianolic acids: small compounds with multiple mechanisms for cardiovascular protection. Journal of Biomedical Science 2011 18:30 